Research Article

\title{
Deep Learning for Detection of Colonic Polyps from Computed Tomography Colonoscopy Images Combined with Colonoscopy
}

\author{
Xiangyan Guo $\mathbb{D}^{1},{ }^{1}$ Hui Gao $\mathbb{D}^{1},{ }^{1}$ Xiaofang Sun $\mathbb{D}^{2},{ }^{2}$ and Surong Li ${ }^{3}{ }^{3}$ \\ ${ }^{1}$ Department of Emergency, Xingtai People's Hospital, Xingtai 054000, Hebei Province, China \\ ${ }^{2}$ Department of Endoscopy, Xingtai People's Hospital, Xingtai 054000, Hebei Province, China \\ ${ }^{3}$ Department of Anesthesiology, Xingtai People's Hospital, Xingtai 054000, Hebei Province, China
}

Correspondence should be addressed to Hui Gao; 14020123@zjnu.edu.cn

Received 7 June 2021; Revised 28 June 2021; Accepted 12 July 2021; Published 20 July 2021

Academic Editor: Gustavo Ramirez

Copyright ( $\odot 2021$ Xiangyan Guo et al. This is an open access article distributed under the Creative Commons Attribution License, which permits unrestricted use, distribution, and reproduction in any medium, provided the original work is properly cited.

\begin{abstract}
The objective of this study was to investigate the diagnosis of colonic polyps (CP) through the computed tomography (CT) images combined with colonoscopy based on Fourier central slice theorem algorithm. In this study, 86 patients with CP admitted to hospital were selected as research objects. CT imaging and colonoscopy were applied to diagnose the patients based on the algorithm of Fourier central slice theorem. The results showed that the diagnostic detection rates of CP and colon cancer (CC) were $88.2 \%$ and $94.2 \%$, respectively. The occurrence site of $\mathrm{CP}$ was the sigmoid and ascending colon. 38 patients were positive for serosal invasion of $\mathrm{CP}$ while 42 patients were negative for serosal invasion of $\mathrm{CP}$, and there were no statistical differences $(P>0.05)$. The lesion positions of remaining 6 cases were hard to find and could not be detected accurately. Besides, the diagnostic accuracy of preoperative and postoperative stages III and IV was all $100.00 \%$. The combination of CT imaging and colonoscopy was employed to diagnose $\mathrm{CP}$, which was found to be able to accurately locate the lesions, to effectively evaluate the tumor stage before and after surgery, and to have a good diagnostic efficacy in detecting tumor serosal layer.
\end{abstract}

\section{Introduction}

$\mathrm{CP}$ refers to all projecting vegetation into the colon lumen, including neoplastic and nonneoplastic vegetation [1]. Neoplastic vegetation has a close relationship with the emerging of cancer and is a precancerous lesion, while nonneoplastic vegetation has little correlation with the emerging of cancer [2]. CP is a common disease in clinic, and its onset age is about 50 years in China. Studies have pointed out that long-term consumption of low-fiber and high-fat foods will increase the incidence of CP [3], and familial genetic factor is also one of the risk factors for $\mathrm{CP}$ [4]. If CP cannot be diagnosed and treated in a timely manner, severe cases may lead to the development of colon tumors and even CC, seriously affecting the life, health, safety, and prognosis of patients.

$\mathrm{CT}$ is a kind of tomography that applies X-ray, $\gamma$-ray, and ultrasound with high sensitivity to scanning a certain part of the human body and has the features of fast scanning time and clear scanning images [5]. Its imaging principle is to employ energy rays to scan the object, rely on the projected data obtained from the outside of the object, and adopt a specific reconstruction algorithm to present the tomography inside the object in the form of two-dimensional or three-dimensional images [6]. Deep learning is a type of machine learning algorithms that use multiple layers to gradually extract higher-level features from the original input. In the image processing, the lower layer can recognize edges, while the higher layer can recognize parts that are meaningful to humans [7]. In recent years, CT imaging has been widely applied in the medical clinical diagnosis. CT examination has become an indispensable method for the diagnosis of colon in the medicine through CT scanning on the injured parts of human body and analyzing the images to determine the disease situation [8]. The deep learning technology was applied in this study for iterative statistics in order to further optimize CT images. 
Colonoscope is a kind of fiber endoscope commonly applied in clinic [9]. Numbere et al. [10] applied colonoscopy to diagnose $\mathrm{CP}$, which could be inserted retrograde through anus to examine the rectum, sigmoid colon, descending colon, transverse colon, ascending colon, and cecum, so that intestinal lesions could be clearly detected and some intestinal lesions could also be treated. Kentaro et al. [11] found that colonoscopy screening in the early stage was conducive to the early detection of lesions and early treatment, so as to avoid the occurrence of advanced CC. In recent years, it has been confirmed at the molecular level that the occurrence and development of CP are multistage and involve a complex process of multigene changes and gradual accumulation, with the development of molecular biology technology. Colonoscopy can visually detect lesions in the intestinal tract, but not the outside, which can be supplemented by CT imaging scanning.

Based on deep learning technology, CT imaging combined with colonoscopy was adopted to diagnose CP and determine detection rate, tumor serosal invasion, and tumor staging, so as to provide a new diagnostic method for clinical $\mathrm{CP}$ and CC.

\section{Materials and Methods}

2.1. Selection of Research Samples. 86 patients with CP were selected as the research objects, who visited hospital from November 17, 2019, to May 19, 2020. There were 48 males and 38 females, with an average age of $56.48 \pm 16.74$ years. All the patients presented clinical manifestations such as fecal bleeding and abdominal pain in the right lower side. The Medical Ethics Committee of hospital had approved this experiment. Each patient and his/her family members had been informed of the experiment and signed the informed consent.

The criteria for inclusion were defined to include patients who conformed to the clinicopathological diagnostic criteria for $\mathrm{CP}$, did not suffer from the contraindications of CT scanning, did not receive surgical treatment, were $18-70$ years old, and had clear consciousness to be examined normally.

The criteria for exclusion were defined to include patients who suffered from hepatic insufficiency, hypertension, and coronary heart disease, had other malignant tumors, had incomplete clinical data, and suffered from mental illness or lack of cooperation.

2.2. Observation Sites and Colonic Polyp Staging. In order to analyze the pathological features of 86 patients in this study, CT imaging was employed to locate $\mathrm{CP}$ and observe the serosal invasion and tumor staging of CP. The main sites of localization were sigmoid colon, ascending colon, descending colon, and cecum. CT diagnosis of CP staging was as follows. First one was stage I for tumor infiltration that occurred only in the region of the third layer of the intestinal wall. Second one was stage II, presenting that infiltrating tumor lesions invaded the fourth layer of intestinal wall. Third one was stage III, showing that tumor lesion invaded the fifth layer structure of intestinal wall and connective tissue around intestines. Fourth one was stage IV that meant that tumor lesions not only violated the full-layer structure of intestinal wall but also infiltrated other peripheral organs. What's more, the clinical and pathological staging of CP was as follows. The thickness of intestinal wall was normal in stage I, and there were internal polyp lesions. At stage II, there was diffuse thickening in the thickness of intestinal wall; the mass appeared inside the intestines, but the external intestinal wall was not infiltrated. The intestinal wall was thickened at stage III, and the mass inside the intestines infiltrated the organs around colon. The lesions at stage IV were transferred into the liver, kidney, bone, and uterus.

2.3. Diagnostic Methods of Colonic Polyps. Colonoscopy had irreplaceable clinical application value in the diagnosis of $\mathrm{CP}$, and it was adopted to directly observe all the colon lumen and intestinal wall, determine the location and size of the tumor, make a preliminary judgment on the infiltration scope, and confirm the diagnosis by biopsy. Early CP in colonoscopy were mainly manifested as the flat and eminence types. The mucosal staining under colonoscopy could detect mild lesions and improve the probability of diagnosis of lesions with flat type. Moreover, the combination of magnified staining colonoscopy and pit pattern classification could further evaluate the depth of infiltration and the nature of lesions. Endoscopic ultrasonography could enhance the evaluation of the infiltration depth of CC, accurately determine the $T$ stage of $\mathrm{CC}$, and play an auxiliary role in the endoscopic method for treatment. Under the influence of its own factors, electronic colonoscopy could only show the lesions inside the colonic cavity but could not observe the lesions outside the colonic cavity, and the examination was invasive and not easy to be accepted by patients.

CT examination was applied with the combination of simulation endoscopy and CT three-dimensional reconstruction technology to evaluate $\mathrm{CP}$, observe the morphology and size of a lesion, and accurately judge the lesion location. In addition, CT examination could reflect the infiltration of tumor outside the intestinal wall, distal organ metastasis, and whether the lymph node was intumescent. Therefore, it had a good effect on analyzing the tumor at the clinical level and diagnosing the tumor staging and could provide a more comprehensive clinical information. Its main features were extremely short duration, being painless, and being noninvasive, and there was a prominent advantage of being able to directly display the lesions and infiltration inside and outside the lumen. Besides, patients had a higher recognition of its existence. At the $T$ stage, a clear boundary between the normal intestinal wall and the lesion site could be found by observing the cross section, and whether the lesion site protruded outside the intestinal cavity or infiltrated the intestinal wall could be visually observed. There was a certain amount of adipose tissue around the intestine. T3 and specific tumor staging evaluation criteria could be defined as fat clearance ambiguity or 
as the presence of strip-like high-density shadow in the fat layer, so a high accuracy could be achieved.

2.4. Computed Tomography Scanning Examination Methods. 86 of the enrolled patients underwent routine CT scans and colonoscopy examinations. In this study, GE Light Speed VCT 64-slice spiral CT scanner (produced by Siemens, Germany) was used for routine CT scanning. During the examination, the supine and prone positions of each patient were taken, respectively, and the scanning range was from the diaphragmatic top to the pubic bone. The scanning parameters were as follows. The layer thickness of scanning was $0.625 \mathrm{~mm}$, the scanning interval was $0.5 \mathrm{~mm}$, the pitch was $0.984: 1$, the matrix was $512 \times 512$, the voltage was $120 \mathrm{kV}$, and the current was $220-500 \mathrm{~mA}$. What's more, the enhancement scanning was adopted by a contrast agent iopromide injection with $300 \mathrm{mg} / \mathrm{mL}$ at an injection speed of $3 \mathrm{~mL} / \mathrm{s}$, with an injection dosage of $1.5 \mathrm{~mL} / \mathrm{kg}$. Oiympuscf240I colonoscopy was used for routine colonoscopy, and patients were examined in turn based on the degree of CP.

After the examination, the CT images were reconstructed and observed by experienced physicians. The site, morphology, and size of the tumor were detected and recorded, and the patients were evaluated according to the type of disease and tumor-node-metastasis (TNM) cancer staging. Then, the patients were examined based on the surgical pathology, and the disease type and TNM staging of the patients were finally determined.

2.5. Fourier Central Slice Theorem Algorithm. Deep learning had achieved remarkable results in the computer vision. It was combined with CT reconstruction in this study, and the times of iterations were counted and reconstruction was analyzed from postprocessing in sequence, so as to propose a new algorithm of Fourier central slice theorem for denoising structure.

The Fourier central slice theorem in two-dimensional images could be expressed as follows. The Fourier transform $Q(\omega)$ of the one-dimensional parallel projection function $q(t)$ of the object density function $g(x, y)$ at a certain angle $\alpha$ was equal to a line of the density function $g(x, y)$ at the same angle in the Fourier space $G\left(\omega_{x}, \omega_{y}\right)$. Moreover, this line was parallel to the detector and went through the origin.

Assuming that the coordinate system of rotation angle $\alpha$ was mon coordinate system, the expression of the density function was $g_{\text {density }}(m, n)$. According to the geometry, it could be determined that mon coordinate system and xoy coordinate system meet the following relations:

$$
\begin{aligned}
& m=x \cos \alpha+y \sin \alpha, \\
& n=-x \sin \alpha+y \cos \alpha .
\end{aligned}
$$

In equations (1) and (2), $\alpha$ stood for the rotation angle, $m$ represented the x-coordinate after rotation, and $n$ expressed the $y$-coordinate after rotation. The parallel projection function was the line integral of the density function $g_{\text {density }}(m, n)$ on the $d$ axis, and it could be expressed as follows:

$$
q(t, \alpha)=(m, \alpha)=\int_{-\infty}^{+\infty} \int_{\text {density }}(m, n) \mathrm{d} n .
$$

In equation (3), $\alpha, m$, and $n$ stood for the rotation angle, the $x$-coordinate after rotation, and the $y$-coordinate after rotation, respectively. The Fourier equation for variable $t$ in the projection equation was transformed into the following:

$$
Q(\omega, \alpha)=\int_{-\infty}^{+\infty} \int_{-\infty}^{+\infty} g_{\text {density }}(m, n) e^{-i 2 \pi \omega c} \mathrm{~d} n \mathrm{~d} m
$$

In equation (4), $\omega$ represented the variables in the Fourier equation. According to equation (1), the following equation can be obtained:

$$
\mathrm{d} n \mathrm{~d} m=\left|\begin{array}{cc}
\partial m \partial x & \partial m \partial y \\
\partial n \partial x & \partial n \partial y
\end{array}\right| \mathrm{d} x \mathrm{~d} y=\mathrm{d} x \mathrm{~d} y .
$$

By substituting equations (5) into (4), equation (6) can be obtained:

$$
Q(\omega, \alpha)=\int_{-\infty}^{+\infty} \int_{-\infty}^{+\infty} g(x, y) e^{-i 2 \pi \omega(x \cos \alpha+y \sin \alpha)} \mathrm{d} x \mathrm{~d} y .
$$

By applying the Fourier transform to the original function, equation (7) could be expressed as follows:

$$
H(a, b)=\int_{-\infty}^{+\infty} \int_{-\infty}^{+\infty} g(x, y) e^{-i 2 \pi(a x+b y)} \mathrm{d} x \mathrm{~d} y .
$$

By comparing equation (4) with equation (5), the following could be obtained:

$$
H(\omega \cos \alpha, \omega \sin \alpha)=Q(\omega, \alpha) .
$$

In equation (8), $a=\omega \cos \alpha$ and $b=\omega \sin \alpha$ in the Fourier space were equivalent to a line that went through the origin and was $\alpha$-angular to the $a$ axis.

2.6. Statistical Methods. In this study, SPSS20.0 statistical analysis software was used for data processing, and percentage (\%) was used for calculation data. The detection rate, specificity, sensitivity, and diagnostic rate were compared through analysis of variance (ANOVA), and the difference was statistically obvious with $P<0.05$.

\section{Results}

3.1. Computed Tomography Imaging Features of Colonic Polyps from Patients. The pathology of $\mathrm{CP}$ was mostly tubular adenoma, its size varied from a few millimeters to a few centimeters, and it was abundant and dense (the number was in 300-3,000 but patients with less than 300 CPs were very few). The predilection sites were more in the left colon, less in the right colon, and much less in the distal ileum. Patients often saw a doctor for hematochezia, mucous stool, anemia, or weight loss. CP could be clearly observed as a rounded mass with sharp edges, while the pedicled polyp was 
presented as a mushroom. CT imaging could visually show polyps protruding into the intestinal lumen (Figure 1).

The pathological features of $\mathrm{CC}$ were as follows. The irregular soft tissue mass shadow appeared in the colonic cavity and was obviously enhanced. The local tube wall was thickened, lumen was narrow, serosal surface was rough, density of fat space around increased slightly, and no obvious enlarged lymph nodes were observed. Ascending colon and small intestine were for diffuse dilatation, and there was fluid accumulation, gas accumulation, and visible gas-liquid level. The best imaging manifestation was localized thickening of the lumen wall of the colon on CT. The normal wall thickness was less than $3 \mathrm{~mm}$, so the wall thickness over $6 \mathrm{~mm}$ was obviously abnormal. If the tumor was confined to the lumen, the edges of the intestine were sharp. However, the edge was irregular if there was parenteral extension in tumor. The soft tissue line extended into the surrounding fat, lesions after enhancement were obviously enhanced, and the growth of mass in the cavity led to narrow lumen, thereby causing the intestinal obstruction. It indicated that mass from the mucous membrane to the cavity grew and infiltrated every layer of intestinal wall, which was suggested to be malignant space-occupying lesion. In addition, advanced cancer could result in annular narrow-apple core lesions of tube wall (Figure 2).

3.2. Detection Rate of Colonic Polyps in Patients. After medical pathological examination, there were 17 patients with CP and 69 patients with CC in this study. CT combined with colonoscopy was employed to diagnose the patients, and it was found that 15 patients were diagnosed as $\mathrm{CP}$ and 65 patients were diagnosed as CC. CP was not easy to be distinguished in clinic, so its detection rate was slightly lower than that of CC. Figure 3 shows the detection rates of CP and CC.

3.3. Localization and Diagnosis for Colonic Polyps of Patients. CT imaging combined with colonoscopy was employed to diagnose CP of the patients, and the main occurrence sites included the sigmoid colon, ascending colon, descending colon, and cecum. The specific detected cases are shown in Figure 4 . The results revealed that $\mathrm{CP}$ mainly occurred in sigmoid and ascending colon.

3.4. Diagnosis of Serosal Invasion of Colonic Polyps. Colonoscopy could display clearly the lesion area and the condition of the proximal and distal colon, as well as observing the lesions of the patient. CT imaging could not only observe the location, morphology, and size of the lesion, but also detect the infiltration of tumors outside the serous membrane. The serosal layer of a tumor inhibited the spread of cancer cells to the periphery. Once a tumor cell broke through the serosal layer, it metastasized to blood vessels, lymph nodes, and surrounding tissues. As shown in Figure 5, 38 patients were positive and 42 were negative, with no statistically substantial difference $(P>0.05)$. In addition, the lesion positions of remaining 6 cases were quite hidden and could not be detected accurately. The specificity, sensitivity, and accuracy of CT examination for CP invasion are shown in Figure 6.

3.5. Diagnosis of Patients with Colonic Polyps before Surgery. Before surgery, patients went through pathological staging, including 16 patients with stage I, 14 patients with stage II, 13 patients with stage III, and 10 patients with stage IV (Figure 7). CT imaging and colonoscopy were combined to diagnose, and Figure 4 indicates the pathological staging. The positive rate of each stage is displayed in Figure 8, finding that the accuracy rates of diagnosis at stages III and IV were both $100.00 \%$. Therefore, it suggested that the late pathologic features of CP were gradually obvious, and the diagnosis rate was higher than the rate of stages I and II.

3.6. Diagnostic Value of Combined Examination in the Staging of CC. The combination of colonoscopy and CT imaging was applied in the diagnosis of staging. The specificity in stages III and IV was $93.97 \%$ and $83.86 \%$, respectively. The sensitivity in stages III and IV was $97.98 \%$ and $100 \%$ in turn. Thus, the diagnostic staging effect was good, and the specific data are shown in Figures 9 and 10.

3.7. Diagnosis of Recurrence in Patients with Colonic Polyps after Surgery. A total of 27 patients suffered from recurrence after surgery, and the recurrence rate was $39.1 \%$. The pathological staging of recurrence patients included 3 patients at stage I, 7 patients at stage II, 10 patients at stage III, and 7 patients at stage IV. The recurrence results of patients at each stage after surgery are shown in Figure 11, and Figure 12 indicates the diagnostic rates of postoperative recurrence at each stage. Besides, the diagnostic rate of patients at stages III and IV was still $100.00 \%$.

\section{Discussion}

The neoplastic and nonneoplastic vegetation in CP were not easy to be distinguished clinically, which was often used as a preliminary diagnosis of polyps. After CP was diagnosed pathologically, there was classification for the next step [12]. Boraschi and Donati [13] adopted colonoscopy and barium meal for the diagnosis of $\mathrm{CP}$, and it was found that the diagnostic effect of the two was good, but they could not observe the external lesions of the intestinal wall and lymph node metastasis and stage the tumor. Matkowskyj et al. [14] applied imaging ultrasound diagnosis and CT examination that could clearly present the anatomical situation and metastasis of the tissue and evaluate the tumor staging. In this study, CT imaging and colonoscopy were combined to diagnose $\mathrm{CP}$, which could not only observe the lesions on the inner intestinal wall, but also examine lymph node metastasis and evaluate the tumor staging, so as to supplement the above research.

CT imaging is extensively applied in the medicine. In order to reduce X-ray damage to patients, low-dose X-ray is of great significance for CT image reconstruction [15]. 


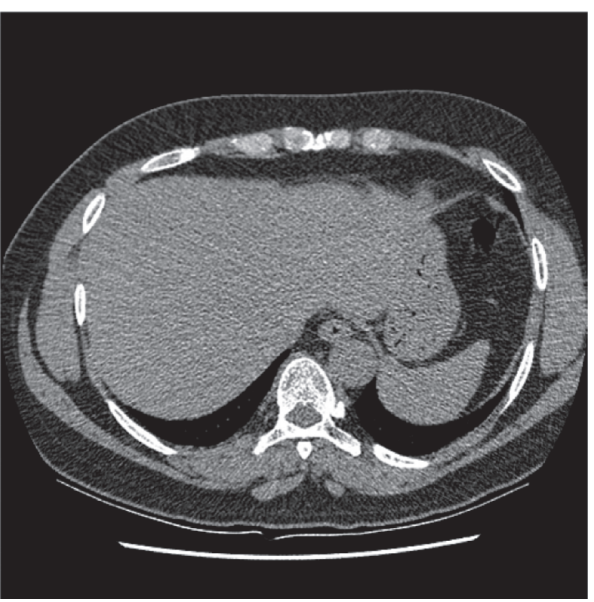

FIGURE 1: CT imaging of CP. There was protrusion to the internal lumen, which was abundant and dense.

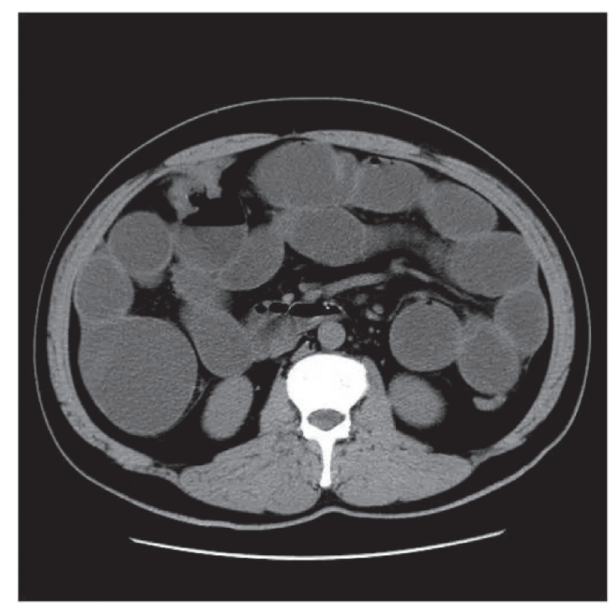

Figure 2: The image of CP cancer. The tube wall was thickened locally and the edge was irregular.

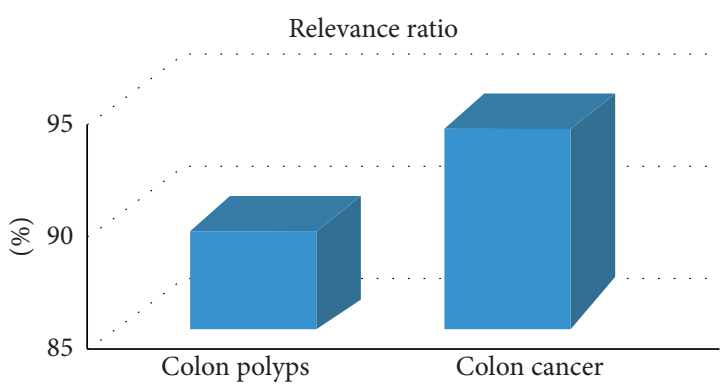

FIGURE 3: Comparison on the relevance ratio of colonic polyps and colon cancer.

Combined with deep learning technology, there was postprocessing, iterative statistics, and improvement and optimization for CT imaging in this study. CT examination is fast and had clear images, which can not only fully display the internal structure of the patient's intestinal cavity, but also analyze the lesions of the patient in real time through the sagittal position angle. In the research of Glissen et al. [16],

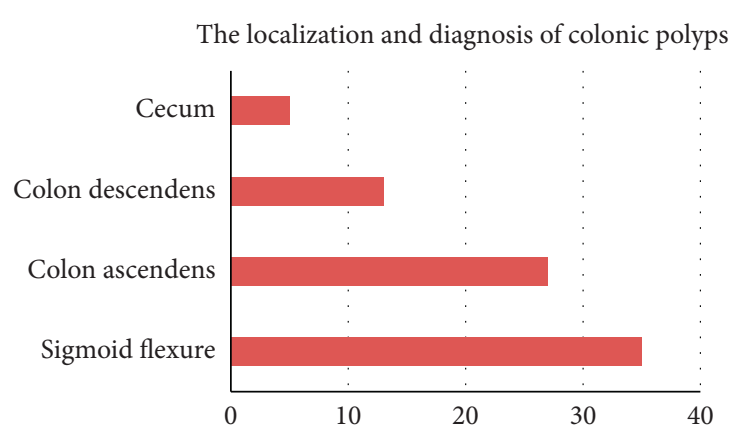

Figure 4: The results of localization and diagnosis of colonic polyps.

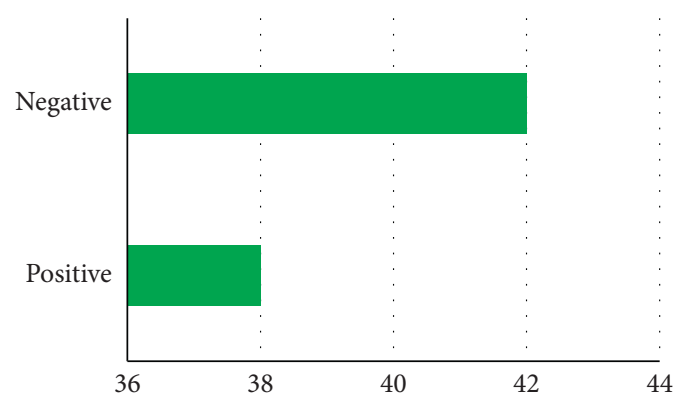

Figure 5: Serosal invasion of colonic polyps. $*$ The difference was statistically substantial in contrast to positive $(P<0.05)$.

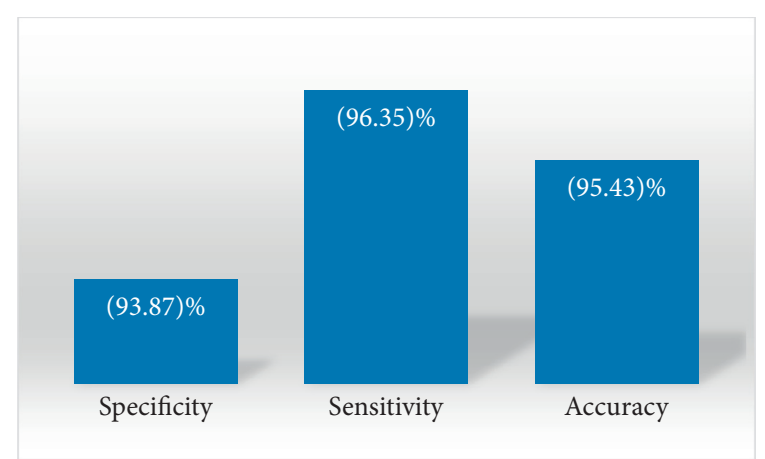

FIgURE 6: Analysis on specificity, sensitivity, and accuracy of CT examination for colonic polyp invasion.

The results of pathological staging

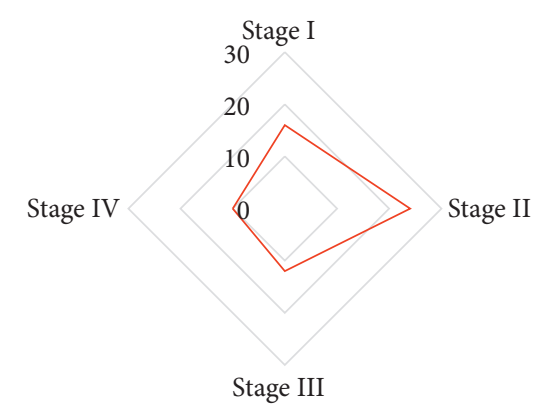

FIGURE 7: The results of pathological staging. 


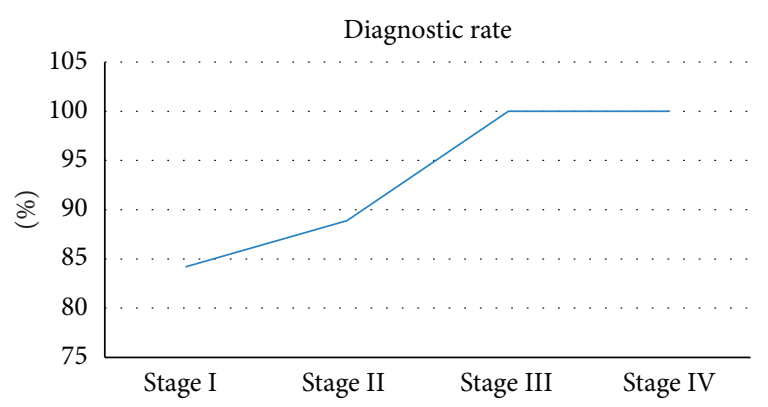

Figure 8: Diagnostic rate of patients at each stage of combined diagnosis.

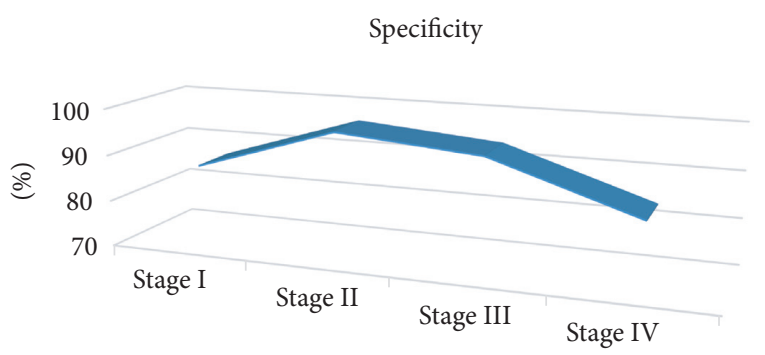

FIGURE 9: Analysis on staging specificity of colonic polyps by CT examination.

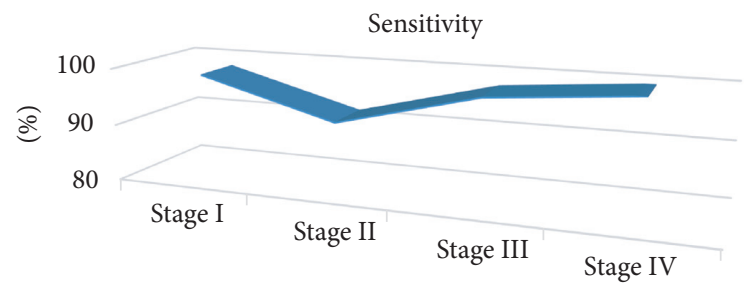

FIGURe 10: Analysis on staging sensitivity of colonic polyps by CT examination.

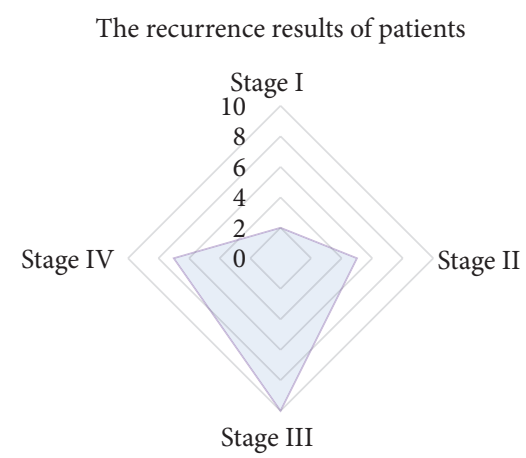

FIGURE 11: The recurrence results of patients at each stage after surgery.

the detection rates of CP and CC were $89.7 \%$ and $93.8 \%$, respectively. The detection rates of $\mathrm{CP}$ and $\mathrm{CC}$ in this study were $88.2 \%$ and $94.2 \%$ in turn, with no marked difference in diagnosis. Abu-Baker et al. [17] found that CP occurred mainly in sigmoid and ascending colon, which was consistent with the positioning results of this study. CT imaging

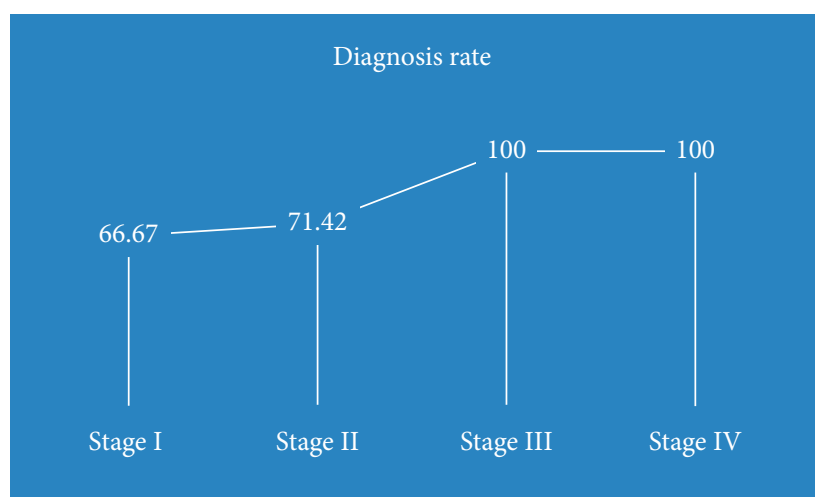

FIGURE 12: Diagnosis rate of postoperative recurrence at each stage.

combined with colonoscopy could reflect the size, morphology, and location of the lesions and could be observed in the infiltration condition of external serosal tumor. Furthermore, its specificity and sensitivity were higher at stages III and IV and it had great diagnostic staging effect in the diagnosis of tumor staging.

To sum up, the evaluation method of CT examination combined with colonoscopy could comprehensively diagnose CP and had good diagnostic effect in the aspects of lesion location, preoperative and postoperative tumor staging, and serosal invasion, which was of great significance for the clinical diagnosis of $\mathrm{CP}$ and CC.

\section{Conclusion}

Based on the deep learning technology, the application of CT imaging and colonoscopy was employed to diagnose $\mathrm{CP}$, indicating that the method could precisely position the lesions. Moreover, it could effectively evaluate the tumor staging for the patient before surgery and the recurrence after surgery, and the staging in stages III and IV had a good effect. Besides, it also had a good diagnosis performance in terms of detection of tumor serosal layer. The limitation of this study is that the combined examination cannot guarantee complete accuracy for small or occult lesions in the colon. In summary, the combination of CT imaging and colonoscopy in this study has good clinical application value.

\section{Data Availability}

No data were used to support this study.

\section{Conflicts of Interest}

The authors declare that they have no conflicts of interest.

\section{References}

[1] J. E. East, W. S. Atkin, A. C. Bateman et al., "British society of gastroenterology position statement on serrated polyps in the colon and rectum," Gut, vol. 66, no. 7, pp. 1181-1196, 2017.

[2] P. Fusaroli, M. Serrani, R. De Giorgio et al., "Contrast harmonic-endoscopic ultrasound is useful to identify neoplastic features of pancreatic cysts (with videos)," Pancreas, vol. 45, no. 2, pp. 265-268, 2016. 
[3] B.-R. Jin, K.-S. Chung, M. Lee, and H.-J. An, "High-fat diet propelled AOM/DSS-induced colitis-associated colon cancer alleviated by administration of Aster glehni via STAT3 signaling pathway," Biology, vol. 9, no. 2, p. 24, 2020.

[4] R. Nallathambi, M. Mazuz, D. Namdar et al., "Identification of synergistic interaction between cannabis-derived compounds for cytotoxic activity in colorectal cancer cell lines and colon polyps that induces apoptosis-related cell death and distinct gene expression," Cannabis and Cannabinoid Research, vol. 3, no. 1, pp. 120-135, 2018.

[5] K. Taguchi, "Energy-sensitive photon counting detector-based X-ray computed tomography," Radiological Physics and Technology, vol. 10, no. 1, pp. 8-22, 2017.

[6] K. H. Dekker, J. J. Battista, and K. J. Jordan, "Stray light in cone beam optical computed tomography: II. reduction using a convergent light source," Physics in Medicine and Biology, vol. 61, no. 7, pp. 2910-2925, 2016.

[7] D. Li, D. Wang, J. Dong et al., "False-negative results of realtime reverse-transcriptase polymerase chain reaction for severe acute respiratory syndrome coronavirus 2: role of deeplearning-based ct diagnosis and insights from two cases," Korean Journal of Radiology, vol. 21, no. 4, pp. 505-508, 2020.

[8] P. Wang, C.-Z. Zhang, G.-B. Wang et al., "Evaluation of computed tomography vascular reconstruction for the localization diagnosis of perigastric mass," Medicine (Baltimore), vol. 97, no. 26, Article ID e11177, 2018.

[9] M. Oda, H. Kondo, T. Kitasaka et al., "Robust colonoscope tracking method for colon deformations utilizing coarse-tofine correspondence findings," International Journal of Computer Assisted Radiology and Surgery, vol. 12, no. 1, pp. 39-50, 2017.

[10] N. Numbere, A. R. Huber, C. Shi, J. M. M. Cates, and R. S. Gonzalez, "Should Ki67 immunohistochemistry be performed on all lesions in multifocal small intestinal neuroendocrine tumours?" Histopathology, vol. 74, no. 3, pp. 424-429, 2019.

[11] I. Kentaro, "Colorectal cancers: an update on their molecular pathology," Cancers, vol. 10, no. 1, p. 26, 2018.

[12] C. R. Lindholm, J. C. Anderson, and A. Srivastava, "The dark side of the colon," Current Opinion in Gastroenterology, vol. 35, no. 1, pp. 34-41, 2019.

[13] P. Boraschi and F. Donati, "MR colonography with a fecal tagging technique and water-based enema for the assessment of inflammatory bowel disease," Japanese Journal of Radiology, vol. 34, no. 8, pp. 585-594, 2016.

[14] N. Horvat, A. Raj, S. Liu et al., "CT colonography in preoperative staging of colon cancer: evaluation of FOxTROT inclusion criteria for neoadjuvant therapy," American Journal of Roentgenology, vol. 212, no. 1, pp. 94-102, 2019.

[15] Y. Zhang, M. C. Lun, C. Li, and Z. Zhou, "Method for improving the spatial resolution of narrow x-ray beam-based X-ray luminescence computed tomography imaging," Journal of Biomedical Optics, vol. 24, no. 8, pp. 1-11, 2019.

[16] P. Wang, X. Xiao, J. R. Glissen Brown et al., "Development and validation of a deep-learning algorithm for the detection of polyps during colonoscopy," Nature Biomedical Engineering, vol. 2, no. 10, pp. 741-748, 2018.

[17] F. Abu Baker, J. A. Z'cruz De La Garza, M. Amir et al., "Colorectal cancer and polyps in diverticulosis patients: a 10year retrospective study in 13,680 patients," Gastroenterology Research and Practice, vol. 2019, p. 6, Article ID 2507848, 2019. 